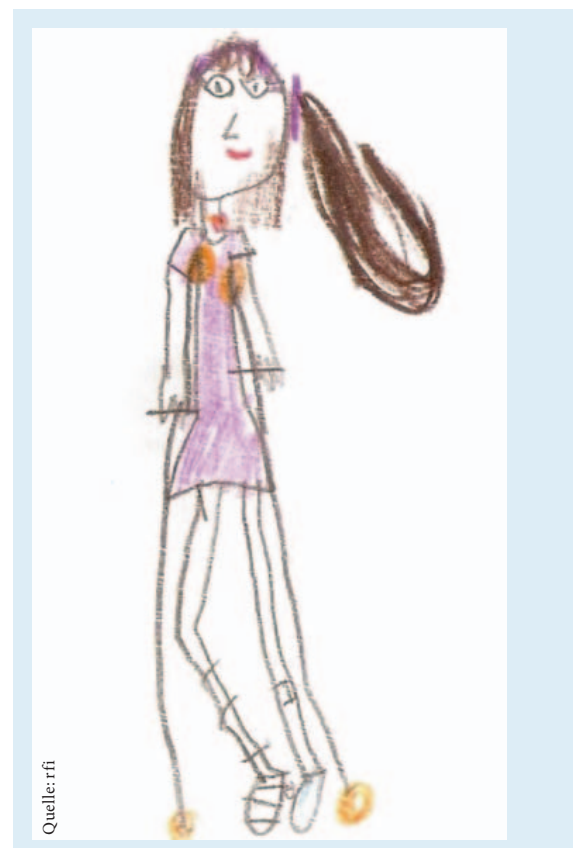

\section{Komplementärmedizin und Pädiatrie}

Den Kindern nur das Beste. Das steht für die meisten Eltern fest; deshalb entscheiden sich viele gegen eine schulmedizinische Therapie, wenn dies ohne Risiken möglich ist. Über den Trend, auf die Kräfte der Natur zu setzen, lesen Sie auf Seite 16. Dieser hat mittlerweile fast alle Industriestaaten erreicht. In der Schweiz, wo etwa die Anthroposophie seit Jahrzehnten ihr Zentrum hat, gibt es eine lange Tradition, in der Pädiatrie einen ganzheitlichen Ansatz zu verfolgen. Was Kinderärzte aus unterschiedlichsten Fachrichtungen über den Einsatz von beispielsweise Homöopathie oder auch TCM denken, lesen Sie in unserem Expertengespräch ab Seite 12. Welche ganzheitlichen Methoden sich eignen, um Kinder zu behandeln, die unter ADHS leiden - je nach Lesart eine Modediagnose oder ein ernst zu nehmendes Gesundheitsproblem - erfahren Sie ebenfalls in dieser Ausgabe.

\title{
Sanfte Methoden für den Zappelphilipp
}

Viele Eltern wollen nicht, dass ihr Kind bei der Diagnose Aufmerksamkeitsdefizit-/Hyperaktivitätsstörung (ADHS) als Therapie Ritalin bekommt. Gefragt sind zunehmend ganzheitliche Behandlungskonzepte, die auf Methoden der Verhaltenstherapie und der Naturheilkunde zurückgreifen. Wunder sollte man sich davon allerdings nicht versprechen.

Ein fast typischer Fall: Als die Erzieherinnen bei Tobias im «kleinen» Kindsgi bemerkten, dass der Junge ziemlich quirlig war, sich nicht auf die Aufgaben konzentrieren konnte und ihnen ziemlich mühsam vorkam, schickten sie ihn zur Abklärung zu einem Kinderarzt. Der lieferte die Diagnose, die immer häufiger gestellt wird, wenn - vor allem bei Buben - das pädagogische Personal eine «Auffälligkeit» bemerkt: ADHS. Die Diagnose hat in den letzten zwei Jahrzehnten so rasant zugenommen, dass heute ein heftiger Streit um die Fragen entstanden ist, ob es sich dabei eigentlich um eine Krankheit handelt oder nicht eher um ein Verhalten, das aufgrund einer veränderten Gesellschaft als pathologisch empfunden wird. Und darum, ob Ritalin das richtige Mittel ist oder ob ganzheitliche Behandlungsmethoden die bessere Alternative sind und dem Kind gerechter werden.

Auf die Biochemie hinter dem ADHS lässt sich derzeit nur durch Indizien schliessen, die die Wirkungsweise der am häufigsten bei der Diagnose eingesetzten Medikamente Ritalin, Concerta oder Medikinet liefern. Alle enthalten den Wirkstoff Methylphenidat, der in den Hirnstoffwechsel eingreift und die Wiederaufnahme von Dopamin hemmt. Dadurch ist Dopamin - über das ADHS-Betroffene scheinbar nicht ausreichend verfügen - für ein paar Stunden wieder in ausreichender Menge verfügbar, sodass die Symptome rasch verschwinden. Die Wirkung erfolgt beinahe schlagartig. Als habe man einen Schalter gekippt, berichten man- che Eltern von ADHS-Kindern. «Die Pille macht fähiger, das Denken ist so konzentriert wie ein Laser», berichtet die Journalistin Birgit Schmid in einer Reportage im Magazin [1] über ihren Selbstversuch mit Ritalin.

Nicht alle Fachleute sind davon überzeugt, dass eine Störung des Hirnstoffwechsels die Ursache von ADHS ist. Den Grund vermutet etwa der deutsche Neurobiologe Gerald Hüther von der Universität Göttingen in Defiziten bei der Sozialisation. Den Kindern fehle die Fähigkeit zur «geteilten Aufmerksamkeit», die andere entwickelten, wenn ihnen der Vater oder die Mutter etwa Büchlein vorläsen oder mit ihnen spielten. Die Therapie müsse darin bestehen, den Kindern zu helfen, die Defizite in ihrer Sozialisation zu beheben.

Gefestigte Kinder sind nicht hyperaktiv Auch andere Experten vermuten, dass das Verhalten der Eltern bei der Entstehung von ADHS eine Rolle spielen könne. Kinder, die nicht die genügende Aufmerksamkeit ihrer Eltern bekämen, so der Erklärungsversuch, versuchten mit Kaspereien und Hyperaktivität das Interesse der Erwachsenen auf sich zu ziehen. «Ein gestärktes Kind mit engen Bindungen an die Eltern, das sich als wichtig erfährt, hat das nicht nötig», sagt Georg Feuser, Professor für Sonderpädagogik an der Universität Bremen. Bei der Diskussion über ADHS stehe immer das Kind als Problem im Mittelpunkt - dabei werde aber die Gesellschaft, in der wir leben, weitgehend ausser Acht gelassen. Zum einen könne sie als Auslöser des ADHS infrage kommen, zum anderen bestim-

\section{KARGER}

Fax +497614520714 


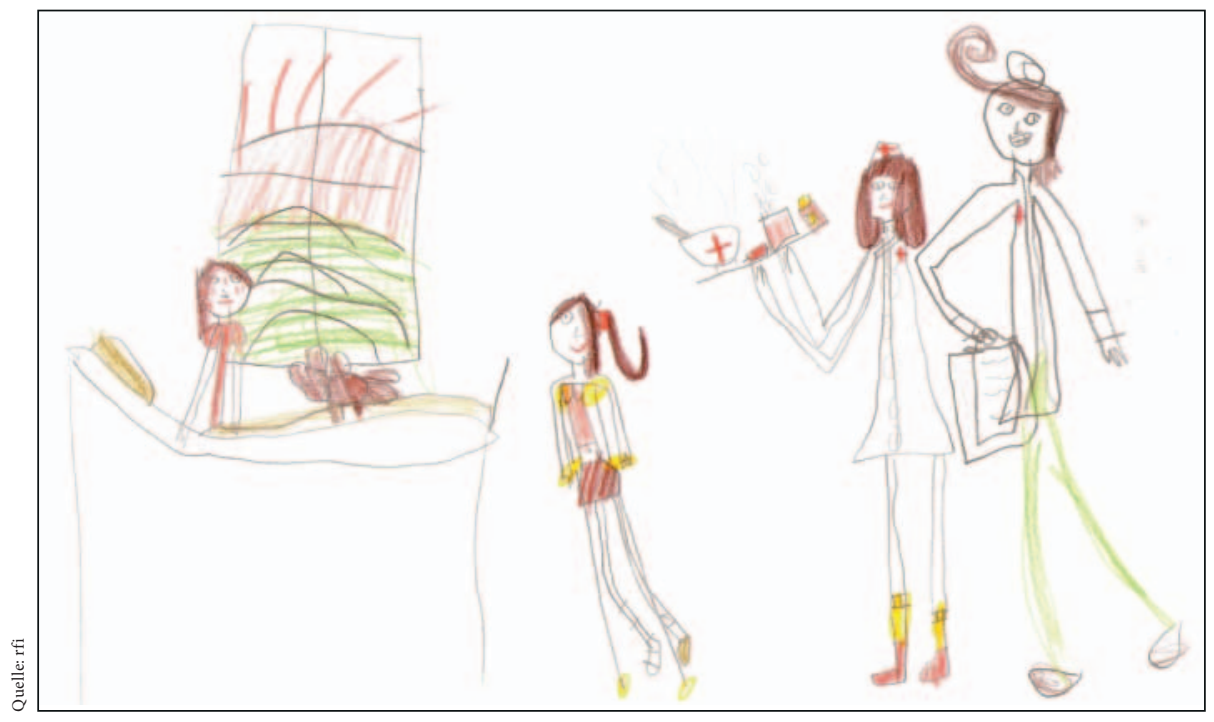

me sie auch, was man als pathologisch empfindet. «Was gestern noch normal war, gilt heute bereits als gestört», meinte etwa der Leiter des Schulpsychologischen Dienstes Baselland Martin Brunner im Beobachterim letzten Frühjahr [2]. Klartext: Heute gehen den chronisch überforderten Lehrerinnen und Lehrern und den gestressten Eltern Kinder viel früher auf die Nerven, als das noch vor zwei oder drei Generationen der Fall gewesen ist.

\section{Konzentration auf das Medikament}

Solche Stimmen sind in der Medizin allerdings in der Minderheit. Von den mehr als 500000 Publikationen, die es mittlerweile über ADHS gibt, beschäftigen sich nur etwa 10000 mit dem psychosozialen Umfeld und nur etwa 10 mit familientherapeutischen Ansätzen, um ADHS zu behandeln. Kein Wunder, dass eine Ritalin-Welle in die Kinderzimmer schwappt. Geradezu explodiert ist in den letzten Jahren der Absatz von Ritalin und ähnlicher Substanzen. Innerhalb eines Jahrzehnts hat er sich verachtfacht.

Die Krankenkasse Helsana hat im vergangenen Jahr Daten über die Häufigkeit der Verschreibung von Ritalin vorgelegt. Aus ihnen geht hervor, dass im Jahr 2005 in der Schweiz 1\% der Schulkinder das Medikament verschrieben bekam; 3 Jahre später waren es bereits 1,7\%. Bei den Buben stieg der Anteil von 1,6 auf 2,7\%. Fachleute die Eltern für das Neurofeedback. Bei dieser relativ neuen Methode sollen Kinder mit ADHS lernen, ihre Gehirnströme zu regulieren und so ruhiger und konzentrierter $\mathrm{zu}$ werden. Mit seiner Mutter besuchte er regelmässig einen Neurofeedback-Trainer. Der befestigte Elektroden, die mit einem Computer verbunden waren, an seinem Kopf. Dann setzte sich der Junge an den Bildschirm, auf dem er nicht etwa den gezackten Verlauf seiner Hirnströme sah, sondern etwas, das eher der Animation eines Computerspiels glich. Die Spielfiguren konnte er mit seinen Hirnströmen steuern. Wenn Tobias es zum Beispiel schaffte, eine Comicfigur allein durch seine Konzentration auf einem Bein zu balancieren, wurde er dafür «belohnt». Dieses positive Feedback soll dafür sorgen, dass Kinder mit ADHS konditioniert werden, sich zu konzentrieren. Mit der Zeit beherrschte Tobias die Methode so gut, dass er sie zu Hause ohne Hilfe des Trainers üben und anwenden konnte. «Neurofeedback ist sehr zeitintensiv», sagt Tobias' Mutter, «allerdings hatten wir damit grossen Erfolg.»

\section{Wirkung ist gut belegt}

Neurofeedback zur Therapie von ADHS ist vergleichsweise gut untersucht, und die Ergebnisse sind ermutigend. In einer vielbeachteten Neurofeedback-Studie [3] in Deutschland, die von der Psychologin Ute Strehl von der Universität Tübingen geleitet wurde, erreichten 60-70\% der Kinder deutliche Behandlungserfolge. In der Schweiz beschäftigt sich die Neuropsychologin Renate Drechsler vom Zentrum für Kinder- und Jugendpsychiatrie (ZKJP) der Universität Zürich in einer interdisziplinären Forschungsgruppe seit langem mit Neurofeedback. In einer Untersuchung [4] verglich sie mit ihrem Team die Wirkung mit derjenigen einer Gruppentherapie; dabei schnitt das Neurofeedback besser ab. Etwa die Hälfte der Probanden hatte gelernt, die Gehirnströme zu regulieren. Drechsler vermutet, dass am Erfolg noch andere Faktoren beteiligt 
gewesen seien. «Die Kinder bekommen ein stärkeres Gefühl der Selbstwirksamkeit», sagt die Neuropsychologin, «sie erhalten die Rückmeldung: Ich habe Kontrolle, ich kann steuern, und das bewirkt etwas. Auch merken die Kinder bewusster, was sie tun.»

Als isolierte Therapiemethode möchte Renate Drechsler das Neurofeedback allerdings nicht propagieren, und auch nicht als Konkurrenz zu Ritalin, wie sie betont. Der Nutzen sei dann am grössten, wenn die Methode in ein «verhaltenstherapeutisches Gesamtkonzept» eingebettet sei. «Das technische Drumherum ist kompliziert, der Übungsaufwand gross», sagt die Neuropsychologin. «Da müssen Eltern und Kinder gleichermassen mitziehen.»

\section{Mit Globuli gegen Nervosität}

Viele Eltern setzen grosse Hoffnung in die Homöopathie. Deren Wirkung bei Kindern mit ADHS wurde in einer KIKOM-Studie [5] in Zusammenarbeit mit dem Berner Kinderarzt Heiner Frei untersucht. Er sieht in ihr eine echte Alternative zu Ritalin und Co. Deren Verbreitung in der heutigen Leistungsgesellschaft schätzt er als "grosses Problem» ein. «Es ist traurig, dass viele Kinder nicht mehr ohne Stimulanzien zurande kommen», sagt er. Das Ergebnis der Untersuchung: Etwa 75\% der Kinder reagieren langfristig befriedigend auf Homöopathie. «Manche können die Behandlung mit der Zeit sogar absetzen», sagt Frei. Nur Kinder mit stärksten ADHS-Symptomen bräuchten Ritalin. Die Berner Langzeitstudie ist die einzige dieser Art weltweit.

Einen schnellen Erfolg darf man sich von der Homöopathie allerdings nicht versprechen. Bis unter den vielen homöopathischen Mitteln, die prinzipiell für ADHS geeignet sind, das individuell richtige gefunden ist und eine Besserung eintritt, vergeht im Schnitt ein halbes Jahr.

Auch Tobias' Eltern unternahmen bei ihrem Sohn einen Versuch mit Homöopathie, allerdings ohne Erfolg. Ein halbes Jahr lang probierten sie unter ärztlicher Anleitung verschiedene Mit- tel aus, doch keines schlug an. Und auch mit Neurofeedback kommt die Familie des inzwischen Neunjährigen an ihre Grenzen. «Wir sind jetzt an einem Punkt, an dem wir überlegen müssen, wie es weitergeht», erzählt die Mutter. Beim Neurofeedback würde vor allem die zeitliche Belastung allmählich zu gross, sagt sie. «Er soll ja auch noch Kind sein dürfen.» (rfi)

\section{Literatur}

1 Schmid B: 10 Milligramm Arbeitswut. Ritalin ist die Modepille der Leistungsgesellschaft. Ein Selbstversuch. Das Magazin. 14.8.2009. dasmagazin.ch/index.php/10-milligramm arbeitswut.

2 Broder S: Ritalin kann ein Segen sein. Beobachter 9/2010.

3 Arns M, de Ridder S, Strehl U, Breteler M, Coenen A: Efficacy of neurofeedback treatment in ADHD: the effects on inattention, impulsivity and hyperactivity: a meta-analysis. Clin EEG Neurosci 2009;40:180-189.

4 Drechsler R, Straub M, Doehnert M, Heinrich $\mathrm{H}$, Steinhausen HC, Brandeis D: Controlled evaluation of a neurofeedback training of slow cortical potentials in children with Attention Deficit/Hyperactivity Disorder (ADHD). Behav Brain Funct 2007;3:35.

5 Frei H: Die homöopathische Behandlung von Kindern mit ADS/ADHS. ZKH 2006;50:5-12.

6 Schulz V: Johanniskraut bei ADHS. Pilotstudie mit mangelbehaftetem Extrakt verfehlt Wirksamkeitsbeweis. Z Phytother 2008;29:280-281.

7 Frei H: Ginkgo biloba bei hyperaktiven Kindern: OffeneStudiezum Nachweis der Wirksamkeitund Verträglichkeit eines pflanzlichen Arzneimittels. Schweiz Zschr Ganzheitsmedizin 2002;14. 10-13.

Niederhofer H: Hopfen scheint einige Symptome der Aufmerksamkeitsdefizit-Hyperaktivitätsstörung (ADHS) zu verbessern. Z Phytother 2009;30:176-177.

9 Pelsser LMJ, Frankena K, Toorman J, Savelkoul HFJ, Pereira RR, Buitelaar JK: A randomised controlled trial into the effects of food on ADHD. Eur Child Adolesc Psychiatry 2008;18:12-19.

\section{Komplementärmedizinische Behandlungsmöglichkeiten}

Es gibt eine Reihe von Studien, bei denen die Wirkung von Phytotherapie auf Kinder mit ADHS getestet wurde. Zum Teil führten sie zu einem negativen Ergebnis, wie etwa im Fall einer randomisierten Doppelblindstudie mit Johanniskrautextrakt, die 2008 in der Zeitschrift für Phytotherapie [6] veröffentlicht wurde. Nach einer Behandlung von 8 Wochen konnte kein Unterschied zwischen der Placebogruppe und der Gruppe, die das Extrakt bekommen hatte, gefunden werden. In einer anderen Untersuchung aus dem Jahr 2002 [7] wurde Ginkgo an 50 Kindern getestet. Von denen sprachen 28, also 56\%, auf das Präparat an. Die Wirkung war aber schwächer als bei Ritalin, und bei deutlich weniger Kindern liess sich ein Nachlassen der Symptome beobachten.

Für verschiedene Pflanzenwirkstoffe gibt es Berichte, nach denen sie in Einzelfällen wirken können. So verbesserte sich bei einem 14-Jährigen die ADHS, nachdem er ein Präparat aus Hopfen bekommen hatte [8].

Neben der Phytotherapie werden auch häufig die Akupressur, Kinesiologie oder Kraniosakraltherapie eingesetzt, um Kinder von der Hyperaktivität «herunter zu bringen». Vergleichende Studien, die den Erfolg dieser Methoden belegen, scheint es allerdings nicht zu geben.

Viele Behandlungsansätze von ADHS zielen auf die Ernährung ab. Die Hypothese, die dahinter steht: Das Gehirn ist über Nervenverbindungen und Hormone mit dem Magen-Darm-Trakt verbunden; deshalb kann es bei bestimmten Nahrungsmitteln ähnlich wie bei einer Körperallergie zu Unverträglichkeitsreaktionen im Gehirn, einer Art «Hirnallergie», kommen. Vier Nährstoffe stehen im Verdacht, bei ADHS mitzuwirken: Zucker, E-Stoffe (Lebensmittelzusätze), Gluten und Milcheiweiss. Wenn ADHS-Kinder auf Glace und Schokolade verzichteten und stattdessen auf eine gesunde, ausgewogene Ernährung umgestellt würden, gehe es ihnen bedeutend besser: Das behauptet die Ratgeberliteratur.

Die Forschung zeigt ein anderes Bild: Was ADHS-Kinder im Alltag essen, schlägt sich kaum auf ihr Verhalten nieder. Zu diesem Schluss kommt eine grosse Übersichtsstudie [9]. 